\title{
DECOMPOSITION OF $\alpha$-TOCOPHERYL SPIRODIMER BY ALKALINE SAPONIFICATION
}

\author{
Osamu Igarashi, Mieko Hagino, and Choten InAGaki ${ }^{1}$ \\ Research Institute of Food Chemistry, Ochanomizu University, \\ 1-1, 2-chome, Otsuka, Bunkyo-ku, Tokyo 112
}

(Received June 12, 1973)

\begin{abstract}
An alkaline saponification method containing pyrogallol is usually applied for the determination of tocopherols. Although there are some metabolites of $\alpha$-tocopherol in tissues, it is not yet clear whether they are true metabolites or degradation products from the alkaline saponification. Since it was shown that $\alpha$-tocopheryl spirodimer, a metabolite of $\alpha$-tocopherol, was degraded mainly to a substance positive to EmmerieEngel reagent, the identification of this substance was carried out. As the model compound of $\alpha$-tocopherol ( $\alpha$-T), mainly 2,2,5,7,8-pentamethyl-6-hydroxy-chromane (M) was used. The results obtained showed that spirodimers of $\alpha-\mathrm{T}$ and $\mathrm{M}$ were reduced to the corresponding dihydroxydimers with pyrogallol during the saponification. Therefore, it was suggested that the alkaline saponification method could not be applied to detect the metabolites from $\alpha$-T and related compounds.
\end{abstract}

An alkaline saponification method containing pyrogallol is usually applied for the determination of tocopherols in foods and tissues. On the other hand, it is known that some metabolites of $\alpha$-tocopherol $(\alpha-T)$ are present in tissues-for example, $\alpha$-tocopheryl quinone, $\alpha$-tocopheryl spirodimer, $\alpha$-tocopheryl dihydroxydimer, $\alpha$-tocopheryl trimer. In an attempt to detect the true metabolites of $\alpha$-tocopherol and its related compounds, we studied whether the alkaline saponification method was useful for the detection of these metabolites or not. The results showed that allmost all of $\alpha$-tocopheryl spirodimer ( $\alpha$-T-SD), which was one of the metabolites from $\alpha$-T in mammals, was converted to another product by the alkaline saponification with pyrogallol. This product was positive to Emmerie-Engle reagent, whereas $\alpha$-T-SD was negative to it. Therefore it was suggested that this product had a phenolic hydroxy group in its structure.

This paper is concerned with the identification of this product and with the possibility of the utilization of the alkaline saponification method for the detection of the metabolites from $\alpha$-T and related compounds.

1 五十嵐脩, 萩野美恵子, 稲垣長典 


\section{EXPERIMENTALS}

1) Materials. $\alpha$-Tocopherol was kindly donated by Eisai Co., Ltd. 2,2,5,7,8-Pentamethyl-6-hydroxy-chromane (M): The model compound of $\alpha$-T having the same structure as $\alpha$-T except one 2-side chain is a methyl group instead of $\mathrm{C}_{16} \mathrm{H}_{33}$. This compound was synthetized from trimethyl-hydroquinone and isoprenes by the method of SMITH (1), and gave needle crystals by twice recrystallization from hexane-benzene solution. This compound showed one spot on TLC* and one peak by GLC.**

Spirodimers of $\alpha$-T and M (I).

Spirodimers were prepared from $\alpha$-T or $\mathrm{M}$ by the alkaline ferricyanide oxidation method (2). But in the case of $\mathrm{M}$, the oxidation time was reduced to one-half of the time necessary for $\alpha$-T (3 min). $\alpha$-T Spirodimer ( $\alpha$-T-SD) was purified by silica gel column chromatography and showed only one spot on TLC. $\alpha$-T-SD was a brilliant yellow oil. M Spirodimer (M-SD) was also purified by silica gel column chromatography. M-SD was crystallized from hexane solution as a yellow needle. M-SD gave only one spot on TLC. These SD are positive against Emmerie-Engle reagent.

2) Alkaline saponification. To the solution of $\alpha$-T-SD or M-SD (150 mg) dissolved in $50 \mathrm{ml}$ of ethyl alcohol, pyrogallol $(0.5 \mathrm{~g})$ and $2 \mathrm{ml}$ of $50 \mathrm{wt} \%$ potassium hydroxide solution were added under nitrogen stream. Then, saponification was carried out for $30 \mathrm{~min}$ under refluxing. Each SD solution was treated similarly with either potassium hydroxide or pyrogallol under the same conditions.

3) Isolation and purification of the main decomposition product from $M-S D$ by alkaline saponification. The spirodimer of the model compound $(1.5 \mathrm{~g})$ in ethyl alcohol $(250 \mathrm{ml})$ was treated with $50 \mathrm{wt} \%$ potassium hydroxide solution $(10 \mathrm{ml})$ and pyrogallol $(2.5 \mathrm{~g})$ for $30 \mathrm{~min}$ under refluxing. To the cooled reaction mixture ethyl ether and water were added. The ether layer was removed, washed with water to neutral, and dried with sodium sulfate. After the evaporation of the ether, the reaction product spontaneously crystallized. Recrystallization was carried out by the addition of hexane to its hot benzene solution.

4) Thin-layer chromatography $(T L C)$. TLC was carried out on a silica gel G plate using chloroform, benzene-chloroform mixture (2:1), or benzene as developing solvent. As coloring reagent, concentrated sulfuric acid and Emmerie-Engel reagent were used.

5) Gas chromatography $(G L C)$. GLC was carried out using Shimadzu GC-4B PTF type as follows: $1.5 \%$ SE-30 on chromosorb w, glass column length $4 \times 3,000 \mathrm{~mm}$, column temperature $250{ }^{\circ} \mathrm{C}$, flow rate $50 \mathrm{ml} / \mathrm{min}$, detector FID, detector temperature $285^{\circ} \mathrm{C}$. A sample was injected directly onto the column.

6) Spectroscopy. IR spectra were measured using JASCO model IR-8,

* TLC, thin-layer chromatography.

** GLC, gas chromatography. 

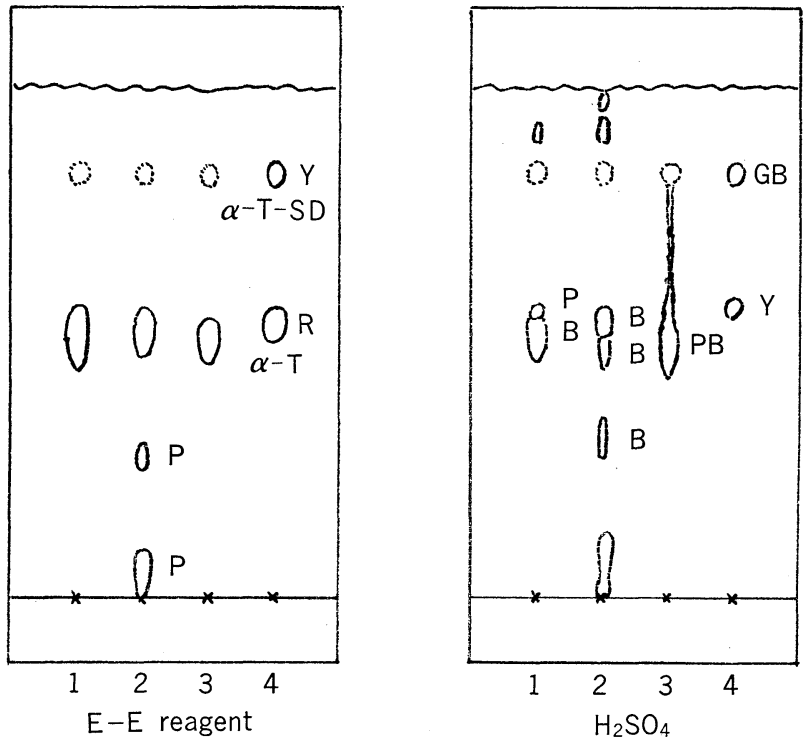

Fig. 1. Thin-layer chromatograms of degradation products from $\alpha$-tocopheryl spirodimer by alkaline saponification. 1) $\mathrm{KOH}$-pyrogallol, 2) $\mathrm{KOH}$, 3) pyrogallol, 4) specimens of $\alpha$-tocopherol and its spirodimer. Y, yellow; R, red; B, brown; P, pink; GB, greyish brown; $\mathrm{PB}$, pinkish brown. Solvent system, $\mathrm{CHCl}_{3}$-benzene $(1: 2)$.

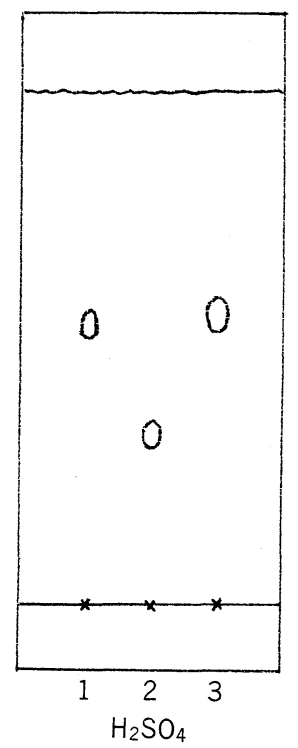

Fig. 2. Thin-layer chromatogram of the main product from spirodimer of $\alpha$-tocopherol model compound. 1) model compound, 2) main product, 3) spirodimer of model compound. Y, yellow; GB, greyish brown; $\mathrm{G}$, grey. Solvent system, $\mathrm{CHCl}_{3}$-benzene $(1: 2)$. 
NMR by JEOL model JNM-3H-100, and MS by Hitachi RMU-6 type.

\section{RESULTS AND DISCUSSION}

Figure 1 shows the thin-layer chromatograms of reaction products of $\alpha$-T-SD treated with (1) KOH-pyrogallol, (2) $\mathrm{KOH}$, and (3) pyrogallol. This figure shows that $\alpha$-T-SD changed to the main product, which had almost as an $R f$ value as that of $\alpha$-T on TLC, by alkaline saponification. And also it suggests that this main product was possibly the same as the main reduction product of $\alpha$-T-SD with pyrogallol. The reaction products of $\alpha$-T-SD had similar IR spectra to that of $\alpha$-T. Figure 2 shows that the main product from M-SD by alkaline saponification had an $R f$ value on TLC different from M. Furthermore, both main reaction products from $\alpha$-T-SD and M-SD by alkaline saponification were positive to EmmerieEngel reagent, unlike $\alpha$-T-SD and M-SD which were negative to this reagent. These results suggest the following: (1) Each main product from $\alpha$-T-SD or M-SD by alkaline saponification is different from the starting material and from the corresponding monomer $(\alpha-\mathrm{T}$ or M). (2) The main products from $\alpha$-T-SD or

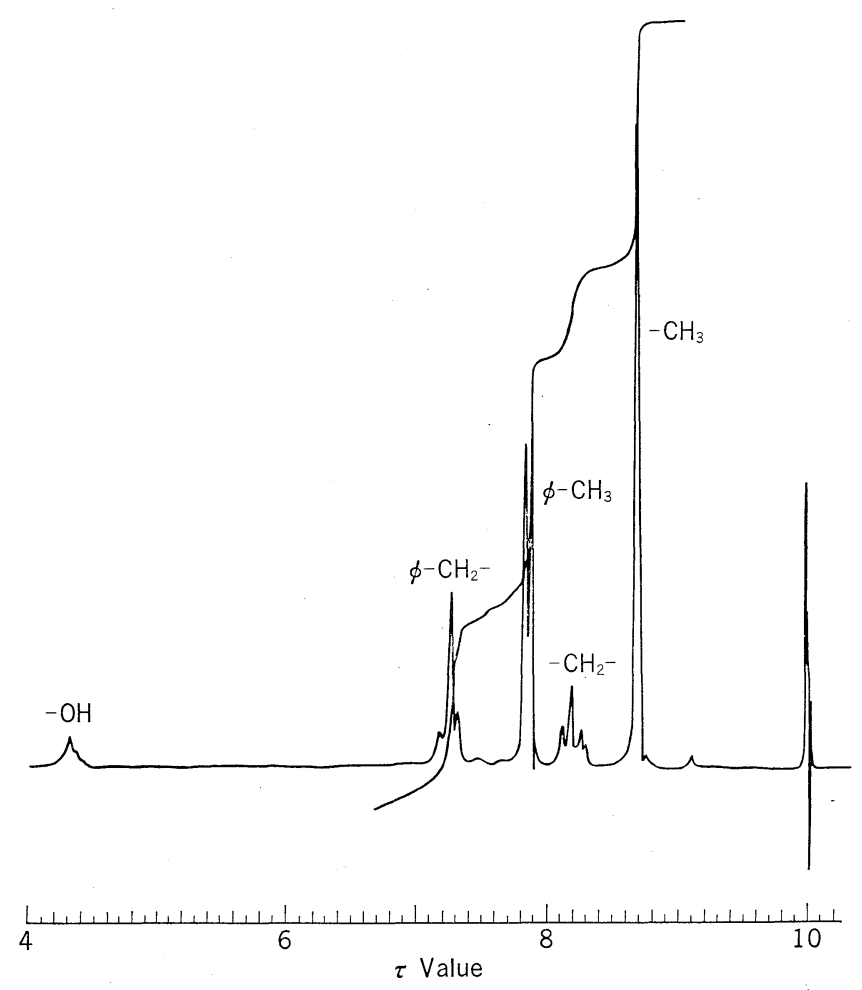

Fig. 3. NMR spectrum of the main product from spirodimer of $\alpha$-tocopherol-model compound. 


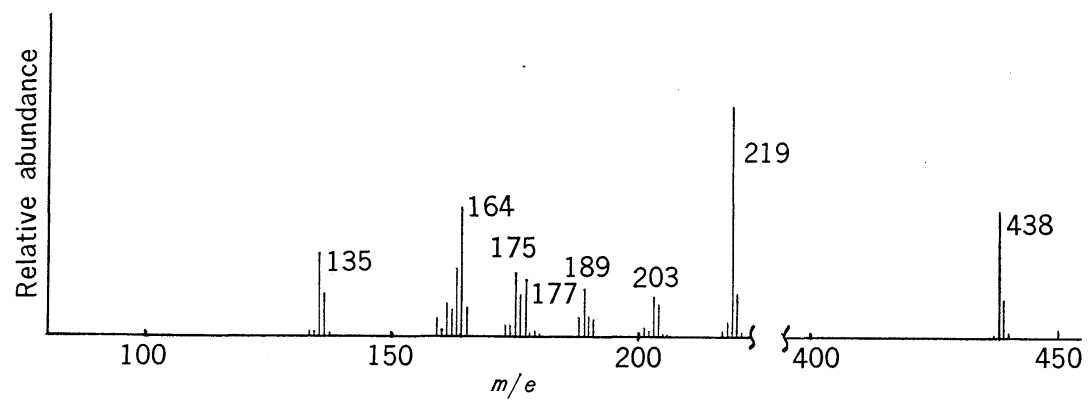

Fig. 4. Mass spectrum of the main product from spirodimer of $\alpha$-tocopherol model compound.

M-SD has a phenolic group in its structure. (3) This products may be produced from $\alpha$-T-SD or M-SD by reduction with pyrogallol.

In order to confirm this suggestion, the isolation and identification of the main product from M-SD by alkaline saponification were examined. The main product was crystallized from ether extract of the reaction mixture and recrystallized, mp 187.5-188 ${ }^{\circ} \mathrm{C}$, Anal: $\mathrm{C}, 76.96 ; \mathrm{H}, 8.62 \%$; Calcd. for $\mathrm{C}_{28} \mathrm{H}_{38} \mathrm{O}_{4}$ (M.W. 438.58) C, 76.67; H, 8.73\%. Mixed mp with authentic specimen of 1,2-di-5'$\left(2^{\prime}, 2^{\prime}, 7^{\prime}, 8^{\prime}\right.$-tetramethyl-6'-hydroxy-) chromanyl-ethane (II, dihydroxydimer of $\mathrm{M}$. mp $184-187^{\circ} \mathrm{C}, \mathrm{C}_{28} \mathrm{H}_{38} \mathrm{O}_{4}$, kindly donated by Professor Fujimaki (3)) was 186$187^{\circ} \mathrm{C}$. Figure 3 shows its NMR spectrum. These peaks were defined as follows: $\tau=8.72\left(12 \mathrm{H},-\mathrm{CH}_{3}\right), 7.90\left(12 \mathrm{H}, \phi-\mathrm{CH}_{3}\right), 8.24\left(4 \mathrm{H},-\mathrm{CH}_{2}-\right), 7.35\left(8 \mathrm{H}, \phi-\mathrm{CH}_{2}-\right)$, $4.32(2 \mathrm{H},-\mathrm{OH})$. As the peak at $\tau=4.32$ disappeared by addition of $\mathrm{D}_{2} \mathrm{O}$, it was defined as an $-\mathrm{OH}$ group.

From the mass spectrum in Fig. 4, its molecular weight was thought to be 348 . The most intensive peak at $m / e=219$ was the half-cleaved ion at the ethane $\mathrm{C}-\mathrm{C}$ bond. These results show that the main product from M-SD by alkaline saponification is a dihydroxydimer of $M$ (II) and that it is produced from M-DS by reduction with pyrogallol as follows:

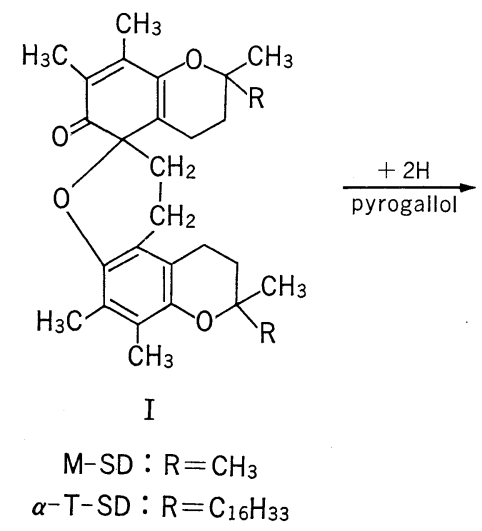<smiles>[R]C1(C)CCc2c(CCCc3c(O)c(C)c(C)c4c3OC([R])(C)CC4)c(O)c(C)c(C)c2O1</smiles>

II 
Therefore, the main product from $\alpha$-T-SD by alkaline saponification must be a dihydroxydimer of $\alpha-\mathrm{T}$ (II, $\mathrm{R}=\mathrm{C}_{16} \mathrm{H}_{33}$ ) the same as in the case of M-SD. This dihydroxydimer of $\alpha$-T will be produced from $\alpha$-T-SD by reduction with pyrogallol. This suggests the possibility that the dihydroxydimer of $\alpha$-T, which is believed to be a metabolite of $\alpha$-T in liver (4), is an artificial product of alkaline saponification. And it also suggests that the alkaline saponification method cannot be applied to experiments on the metabolism of $\alpha-\mathrm{T}$ and related substances. Therefore, a further examination is necessary to detect a dihydroxydimer of $\alpha-\mathrm{T}$ as a true metabolite of $\alpha$-T. Next, since the dihydroxydimer of $\alpha$-T is positive to Emmerie-Engel reagent, the use of Emmerie-Engel reagent should be avoided for the determination of $\alpha$-T in animal tissues, especially in Vitamin E-deficient rats, because Emmerie-Engel colorimetry estimates the value for $\alpha$-T to be excessive and $\alpha$-T is not easily separated from its dihydroxydimer by TLC.

Furthermore, it was investigated whether or not, in the determination of $\alpha$-T by GLC, the interfering substances were produced from $\alpha$-T-SD by alkaline saponification. The results showed that a peak having the same retention time as $\alpha$-T on an SE-30 column was produced from $\alpha$-T-SD by alkaline saponification. But its yield was very small (about $2-3 \%$ for the $\alpha$-T-SD). Then it was suggested that this product did not interfere with the determination of $\alpha$-T, practically. The alkaline saponification method accompanied by GLC is useful for the determination of tocopherols in foods.

The authors wish to express their sincere thanks to Professor Masao Fujimaki, University of Tokyo, for his kindness in donating the dihydroxydimer of $\mathrm{M}$.

\section{REFERENCES}

1) Smith, L. I., Vngnade, H. E., Hoehn, H. H., and Wawzonek, S., J. Org. Chem., 4, 311 (1939).

2) Nelan, D. R. and Robeson, C. D., J. Amer. Chem. Soc., 84, 2963 (1962).

3) Fujimaki, M., Kanamaru, K., Kurata, T., and Igarashi, O., Sgr. Biol. Chem., 34, 1781 (1970).

4) Draper, H. H., Csallany, A. S., and Chiu, M., Lipids, 2 , 47 (1967). 